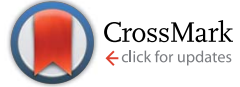

Cite this: RSC Adv., 2016, 6, 11546

Received 30th September 2015

Accepted 13th January 2016

DOI: $10.1039 / c 5 r a 20218 c$

www.rsc.org/advances

\section{Systemically replicated organic and inorganic bony microenvironment for new bone formation generated by a 3D printing technology $\uparrow$}

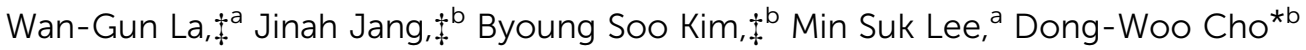 \\ and Hee Seok Yang*a
}

Here, bone demineralized and decellularized extracellular matrix (bdECM) was coated on the surface of the 3D printed polycaprolactone (PCL), poly(lactic-co-glycolic acid) (PLGA), and $\beta$-tricalcium phosphate (TCP) mixture scaffolds (PCL/PLGA/TCP) that induce in vitro osteogenic activity and in vivo critical sized bone defect healing. Collagen coating on PCL/PLGA/TCP was used as a positive control. bdECM coating on $\mathrm{PCL} / \mathrm{PLGA} / \mathrm{TCP}$ successfully proceeded maintaining its pore interconnective structure. PCL/PLGA/TCP/ bdECM showed the highest osteoblast adhesion with alignment morphology. These enhanced cell adhesion and alignment might contribute to in vitro osteogenic activity of osteoblasts combined with osteogenic biomolecules in bdECM. Furthermore, in vivo bone defect healing was enhanced compared to the other scaffolds. Three type of scaffold (PCL/PLGA/TCP, PCL/PLGA/TCP/Col, and PCL/PLGA/TCP/ bdECM) with or without osteoblasts were implanted into the mouse calvarial defect. In vivo bone healing was measured by microcomputed topography and histological staining. PCL/PLGA/TCP with or without osteoblast implantation on the mouse calvarial defect only showed fibrous tissue formation. PCL/PLGA/ $\mathrm{TCP} / \mathrm{Col}$ with osteoblasts showed the half ratio of bone healing. However PCL/PLGA/TCP/bdECM without osteoblasts showed significantly enhanced bone defect healing. This result indicated that PCL/ PLGA/TCP/bdECM is sufficient to induce the critical bone defect healing due to the bdECM containing biomolecules and enhanced host cell ingrowth into the defect.

\section{Introduction}

Extracellular matrix (ECM) provides crucial cues for long-term cell proliferation and differentiation and creates a microenvironment, including cell-to-cell connections and threedimensional (3D) cellular organization. ECM, composed of various collagens, non-collagenous proteins (NCPs) and proteoglycans, is a highly sophisticated biomaterial. ${ }^{\mathbf{1}}$ It is thus difficult if newly developed biomaterials mimic the complex ECM composition of the target tissue. ${ }^{1}$ Among many ECMs, collagen is a preponderant and predominant organic constituent of the bone tissue secreted by osteoblasts. The secreted-collagen forms the dense structural hierarchies by combining with NCPs such as osteocalcin, bone sialoprotein and osteopontin. The bone

\footnotetext{
${ }^{a}$ Department of Nanobiomedical Science \& BK21 PLUS NBM Global Research Center for Regenerative Medicine, Dankook University, Cheonan 330-714, Republic of Korea. E-mail: hsyang@dankook.ac.kr; Fax: +82-41-559-7839; Tel: +82-41-550-1177 ${ }^{b}$ Department of Mechanical Engineering, Pohang University of Science and Technology (POSTECH), Pohang, 790-784, Korea.E-mail:dwcho@postech.ac.kr; Fax: +82-54-2792863; Tel: +82-54-279-2171

$\dagger$ Electronic supplementary information (ESI) available. See DOI: $10.1039 / \mathrm{c} 5 \mathrm{ra} 20218 \mathrm{c}$

\$ These authors equally contributed to this work. D. W. Cho and H. S. Yang has co-corresponded of this work.
}

mineralization was then performed for the hardness and strength of the tissue by transforming amorphous and noncrystalline ECMs into more crystalline forms. ${ }^{2}$ Numerous studies have shown the important role of NCPs for the bone mineralization. ${ }^{3,4}$ Although these proteins contribute a relatively small amount of the total protein mass, they are known as a modulator in various different steps of the bone mineralization, including the formation of the amorphous calcium phosphate, apatite nucleation and crystal growth. ${ }^{4}$

Demineralized bone matrix (DBM) has been developed to recapitulate the microenvironment containing growth factors, collagen and NCPs, and the most common clinical form of DBM is a stable suspension of particles. ${ }^{5}$ Although its osteoinductive effect has been well defined in animal studies, ${ }^{6,7}$ there is insufficient information for human clinical studies despite a strong clinical demand for DBM products. ${ }^{8}$ Moreover, a recent study has shown that DBM produces more inflammatory response than a synthetic hydroxyapatite compound ${ }^{9}$ due to the use of additional viscose carriers such as water-soluble polymers (sodium hyaluronate or carboxymethylcellulose) or anhydrous water-miscible solvents (glycerol). ${ }^{9}$ Demineralized and decellularized bone extracellular matrix (DBM/ECM) was developed to make a gel form of DBM/ECM biological material without the use of additional solvents, and it has been indicated 
that DBM/ECM hydrogel retains both osteoconductivity and osteoinductivity. ${ }^{10}$

3D polymeric scaffold prepared by 3D printing (3DP) technology has the more stable and higher throughput results than those generated by the conventional fabrication techniques in bone tissue engineering. ${ }^{11,12}$ It is considered as an alternative of autologous and allograft substitute because of the major clinical need for versatile biomaterial systems. ${ }^{11}$ In addition, 3DP technology is capable of not only maintaining the repeatable fabrication results, but also reconstitution of the complexity of the defected bone so that it can be applied to develop the patients' specific tailored structure. ${ }^{11,12}$ A major limitation lies in the passive nature of these materials, postulating functionalization through the incorporation of bioactive molecules to bring out desirable biological responses. ${ }^{13,14}$ Some of the previous studies have shown the superiority of $\beta$-tricalcium phosphate ( $\beta$-TCP), a principal inorganic constituent of natural bone and extremely potent for stimulating bone formation, mixed PCL/PLGA scaffold (PCL/PLGA/TCP scaffold) for the regeneration of critical sized rat calvarial defect. ${ }^{12}$ TCP is known to show the most extensive bone remodeling among the bioceramics and allows osteogenesis to occur and form tight bonds with host bone tissues. ${ }^{13}$

Here, we develop a novel bone tissue substitute to systemically replicate the organic and inorganic phases of bony microenvironment. PCL/PLGA/TCP scaffold functions as the structurally supportive inorganic phase and is coated with the $1 \%$ bone demineralized and decellularized ECM (bdECM) solution. The scaffold, fabricated by Multi-Head Deposition System (MHDS) based on the 3D printing technique developed in house, ${ }^{14}$ has fully interconnected pores and sufficient mechanical strength. The bdECM coated PCL/PLGA/TCP scaffolds enhances differentiation and mineralization of seeded osteoblasts in vitro. It also promoted higher bone mineralization within a shorter period than that of the current bone substitute by inducing a robust cellular response in mouse calvarial defects model.

\section{Materials and methods}

\section{Printing of PCL/PLGA/TCP scaffolds}

PCL (MW 43 000-50 000; Polysciences Inc.,Warrington, PA, USA) and PLGA (MW 50 000-75 000; Sigma-Aldrich, St. Louis, MO, USA) were placed in the glass container at $130{ }^{\circ} \mathrm{C}$ for 10 min. The molten polymers were mixed with TCP (SigmaAldrich) powder at a final concentration of $10 \%$. The prepared blend was transferred in a $10 \mathrm{~mL}$ steel syringe of MHDS and dispensed through the precision nozzle under $140{ }^{\circ} \mathrm{C}$ at a pneumatic pressure of $650 \mathrm{kPa}$. Printed scaffold was sterilized in $70 \%$ ethanol for $30 \mathrm{~min}$ and exposed under UV light overnight prior to the coating process.

\section{Bone preparation}

Fresh bovine tibiae were harvested from cattle aged 12-24 months. Bones were received in segmented form and were separated into cancellous and cortical groups, and the cancellous group was used in this study. Bones were either used as received or stored at $-20{ }^{\circ} \mathrm{C}$ to preserve their osteoinductive potential, ${ }^{15}$ and processed using the modification of previously reported methods. ${ }^{2}$ The cancellous segments were cleaned of residual tissue and washed with phosphate-buffered saline (PBS) containing $0.1 \%(\mathrm{w} / \mathrm{v})$ gentamicin (Invitrogen, Carlsbad, CA, USA). The washed segments were frozen in liquid nitrogen and sectioned to produce fragments no greater than $4 \times 4 \times 4$ $\mathrm{mm}^{3}$. Fragments were washed in distilled water, immersed in liquid nitrogen and ground in a commercial coffee mill (Sun Fresh, Kordia Co., Daegu, Gyeongbuk, Korea).

\section{Demineralization and decellularization}

The cancellous bone granules were demineralized using an following previously reported methods. ${ }^{19}$ In brief, the granules were demineralized under agitation in $0.5 \mathrm{~N} \mathrm{HCl}\left(25 \mathrm{~mL} \mathrm{~g}^{-1}\right.$ bone) at room temperature for $24 \mathrm{~h}$. Stirred beakers of bone granules and acid were agitated at $300 \mathrm{rpm}$ to generate a small vortex; particles were suspended in motion in the acid and did not settle during the process. After demineralization the resultant material, referred to as bovine demineralized bone matrix (bDBM), was filtered separated under vacuum from the acid and rinsed with distilled water. The lipid in the demineralized powder was then extracted with a 1:1 mixture of chloroform (Fisher Scientific, Loughborough, UK) and methanol (Fisher Scientific) for $1 \mathrm{~h}$ and then repeatedly rinsed, firstly in methanol and then distilled water. The bDBM was then snapped frozen, lyophilized overnight and stored at $-20{ }^{\circ} \mathrm{C}$ until required. An enzymatic decellularization protocol, adapted from previously reported methods ${ }^{16,17}$ was used. Briefly, the lyophilized bDBM was rinsed with distilled water and decellularized in a solution of $0.05 \%$ trypsin (Sigma-Aldrich) and $0.02 \%$ ethylenediamine tetraacetic acid (EDTA) (Sigma-Aldrich) at $37{ }^{\circ} \mathrm{C}$ and $5 \% \mathrm{CO}_{2}$ under continuous agitation for $24 \mathrm{~h}$. The resultant material, referred to as bovine decellularized matrix (bECM) was rinsed in PBS supplemented with $1 \% \mathrm{w} / \mathrm{v}$ penicillin/streptomycin under continuous agitation for $24 \mathrm{~h}$ at $4{ }^{\circ} \mathrm{C}$ to remove residual cellular material. The bECM was then snap frozen, lyophilized overnight and stored at $-20{ }^{\circ} \mathrm{C}$.

\section{bdECM surface coating on PCL/PLGA/TCP scaffolds}

The bdECM coated PCL/PLGA/TCP scaffold (PCL/PLGA/ $/$-TCP/ bdECM) was produced by the dip-coating method according to the modified protocol form the reference. ${ }^{16,17}$ Briefly, the coating solution was prepared by dissolving bdECM in distilled water at a concentration of $10 \mathrm{mg} \mathrm{mL}^{-1}$. As a comparison, the commercially available atelocollagen solution (Koken, Shizuoka, Japan) was also provided at a $10 \mathrm{mg} \mathrm{mL}^{-1}$ concentration and coated on the PCL/PLGA/TCP scaffold (PCL/PLGA/TCP/Col). The scaffold was centrifuged at $4{ }^{\circ} \mathrm{C}$ to remove the excessive solutions from the porous area of the scaffold and was incubated at $37^{\circ} \mathrm{C}$ for $20 \mathrm{~min}$ followed by the air-dry process under the ambient and sterilized condition air for $20 \mathrm{~min}$. This process was repeated for 3 times. 


\section{Scaffold characteristics}

The morphology of the scaffolds was observed using a scanning electron microscope (SEM; JSM-5300, JEOL, Tokyo, Japan) at $10 \mathrm{kV}$. The scaffolds were coated with platinum using a sputtercoater for $120 \mathrm{~s}$. The surface morphology of the PCL/PLGA/TCP scaffold was compared to that of the PCL/PLGA scaffold. Noncoated PCL/PLGA/TCP scaffold was used as a control group at all the experiments. The degree of the bdECM and collagen coating on the scaffold was demonstrated by spectra of X-ray photoelectron spectroscopy (XPS) against nitrogen (N), oxygen (O), and carbon (C) (ESCALAB 220iXL; VG Scientific, East Grinstead, West Sussex, UK).

\section{In vitro cell culture}

To analyze in vitro cell behavior, the isolated primary osteoblasts were seeded at a density of $1 \times 10^{4}$ cells on each scaffold. Calvarial osteoblasts were isolated from the calvaria of neonatal (less than 1 day old) Sprague-Dawley rats (SLC, Tokyo, Japan) by a digestive enzymatic process. ${ }^{23}$ Cell seeded scaffold was immersed and culture in growth medium for a day to facilitate cell attachment, and then the culture medium was replaced to the osteogenic medium containing $50 \mathrm{mM}$ L-ascorbic acid-2phosphate (Sigma-Aldrich), $10 \mathrm{mM} \quad \beta$-glycerophosphate (Sigma-Aldrich), and $100 \mathrm{nM}$ dexamethasone (Sigma-Aldrich) in complete alpha minimum essential medium to induce the osteogenic function of the osteoblasts. The medium was changed every other day.

\section{Osteoblasts morphology observation}

Calvarial osteoblasts were isolated from the calvaria of neonatal (less than 1 day-old) Sprague Dawley rats (Japan SLC, Tokyo, Japan) by a digestive enzymatic process. The osteoblasts seeded scaffold was harvested on day 1 and 14 to observe the cell morphology on each scaffold via SEM analysis. The harvested scaffolds were fixed in the modified Karnovsky's fixative containing $2 \%$ paraformaldehyde and $2 \%$ glutaraldehyde in $0.05 \mathrm{M}$ sodium cacodylate buffer (SC buffer (pH 7.2); Sigma-Aldrich) at $4{ }^{\circ} \mathrm{C}$ for $4 \mathrm{~h}$. The primary fixed scaffolds washed with $0.05 \mathrm{M} \mathrm{SC}$ buffer at $4{ }^{\circ} \mathrm{C}$ for three times, and the treated scaffolds were then immersed in 1\% osmium tetroxide (Sigma-Aldrich) in 0.05 M SC buffer at $4{ }^{\circ} \mathrm{C}$ for $2 \mathrm{~h}$. The post fixed scaffolds were serially dehydrated and dried with hexamethyldisilazane (SigmaAldrich) for $15 \mathrm{~min}$.

\section{Cell proliferation analysis}

Osteoblasts proliferation on the scaffold was conducted using a Cell Count Kit-8 (CCK-8, Dojindo, Kumamoto, Japan). The scaffold was immersed in the diluted CCK- 8 solution with growth medium (1:10 ratio) and incubated at $37^{\circ} \mathrm{C}$ for $2 \mathrm{~h}$. The solution was then extracted and measured at $450 \mathrm{~nm}$ using a microplate reader (Asys UVM 340; Biochrom, Cambridge, UK). The cell seeded scaffold was washed with PBS and incubated with new culture medium.

\section{Effect of PCL/PLGA/TCP/bdECM scaffold on osteogenic function and bone mineralization}

Osteoblasts cultured scaffold in the osteogenic media were rinsed with PBS and fixed in $4 \%$ paraformaldehyde solution at day 14 . The fixed scaffolds were treated with $2 \%$ bovine serum albumin (BSA) in PBS to block non-specific background and immunostained using primary antibody against ALP antibody (1 : 200; Santa Cruz Biotechnology, Santa Cruz, CA, USA) followed by the incubation with Alexa Fluor 488 goat anti-rabbit antibody (1:100; Invitrogen). All the samples were counterstained with DAPI (1:1000; Sigma-Aldrich) and visualized using a laser scanning confocal microscope (Olympus FluoView $^{\mathrm{TM}}$ FV1000, Tokyo, Japan). ALP activity was also quantified by $p$-nitrophenyl phosphate ( $p$ NPP; Sigma-Aldrich) at day 14. Osteoblasts seeded onto the scaffolds were lysed in RIPA lysis buffer (Millipore, Billerica, MA, USA), and lysates were incubated in ALP substrate buffer containing $5 \mathrm{mM} p \mathrm{NPP}$ at 37 ${ }^{\circ} \mathrm{C}$ for $30 \mathrm{~min}$. The enzymatic activity of ALP was measured at $405 \mathrm{~nm}$ using the microplate reader. The $\mathrm{Ca}^{2+}$ concentration in the lysate was measured spectrophotometrically using the Calcium Detection Kit (Abcam, Cambridge, MA, UK) according to the manufacturer's instructions. At the requisite time period (day 21), cell seeded scaffolds were fixed with $4 \%$ paraformaldehyde to stain calcium deposition. The cells were then stained with $2 \%$ Alizarin Red S (Sigma-Aldrich) solution ( $\mathrm{pH} 4.3$ ) at room temperature (RT) for $20 \mathrm{~min}$. The calcium deposition area was observed under a digital microscope (Dino lite, New Taipei, Taiwan), and the amount of calcium deposition was quantified by extracting the Alizarin Red stain from the cells using dimethyl sulfoxide (Sigma-Aldrich) at RT for $2 \mathrm{~h}$. The extraction was measured at $570 \mathrm{~nm}$ using a microplate reader. Osteogenic differentiation related mRNA expressions of cultured osteoblasts were analyzed through qRT-PCR. Cultured cells were isolated using RNAiso reagent (Takara, Japan) following the manufacturer's protocol at day 21. RNA concentration was measured using Nanodrop (Thermo Scientific, USA), and reverse transcription was performed with an amfiRivert cDNA synthesis platinum master mix kit (GenDEPOT, USA). Gene expression was analyzed with LightCycler SYBR Green I Master mix using LightCycler® 480 Real-Time PCR instrument (Roche Biochemicals, IN, USA). Primer sequences were designed referring to NCBI and PubMed databases and presented in Table S1. $\dagger$

\section{Mouse calvarial defect model for evaluating bone regeneration}

A mouse calvarial defect model was produced as previously described. ${ }^{19}$ Six-week-old mice from the Institute of Cancer Research (Koatech, Sungnam, Kyunggi-do, South Korea) were anesthetized with xylazine $\left(20 \mathrm{mg} \mathrm{kg}^{-1}\right)$ and ketamine $(100 \mathrm{mg}$ $\mathrm{kg}^{-1}$ ). After the scalp hair was shaved, a longitudinal incision was made in the midline of the cranium from the nasal bone to the posterior nuchal line, and the periosteum was elevated to expose the surface of the parietal bones. Using a surgical trephine burr (Ace Surgical Supply Co., Brockton, MA, USA) and a low-speed micromotor, 2 circular transosseous defects 
(diameter, $4 \mathrm{~mm}$ ) per mouse were produced in the skull. The defect size corresponded to the critical defect size for the mouse calvarial defect model. ${ }^{19}$ The drilling site was irrigated with saline, and the bleeding points were electrocauterized. Five animals (10 defects) were used in each group. The animal study was approved by the Institutional Animal Care and Use Committee at Dankook University (DKU-14-012).

\section{Micro-CT analysis}

Eight weeks after implantation, the animals were euthanized by $\mathrm{CO}_{2}$ asphyxiation, and the skulls were harvested for analysis. Bone formation was evaluated with micro-CT scans ( $n=7$ per group). The micro-CT images were obtained using a micro-CT scanner (SkyScan-1172, Skyscan, Kontich, Belgium). The new bone volume was determined using a CT analyzer program (CT-An, Skyscan).

\section{Histological analysis}

After micro-CT imaging, the specimens were prepared for histological and histomorphometric analysis. The specimens were immersed in $10 \%$ (v/v) buffered formalin solution, dehydrated in alcohol solutions of increasing concentrations, clarified in xylene, and embedded in paraffin. One sagittal and one frontal section from each of two specimens per animal were obtained by a microcutting and grinding technique. The sections were stained with Goldner's trichrome stain. The area of bone formation was determined using Adobe Photoshop software (Adobe Systems, Inc., San Jose, CA, USA) by the percentage of newly formed mineralized bone. The percentage of bone formation area in the defect area was calculated as (new bone area/bone defect area) $\times 100$. Bone density was calculated as [new bone area/(new bone area + fibrous tissue area + residual biomaterial area) $] \times 100 .^{20}$

\section{Statistical analysis}

Quantitative data were expressed as the means \pm standard deviations. The statistical analysis was performed using one-way analysis of variance (ANOVA) with the Tukey significant difference post hoc test using SPSS software (SPSS Inc., Chicago, IL, USA). A value of $p<0.05$ was considered to denote statistical significance.

\section{Results and discussions}

\section{Characterization of 3D printed PCL/PLGA/TCP scaffolds and coated scaffolds}

PCL/PLGA/TCP scaffold was generated by dipping in the bdECM and collagen solution and evaluated for both in vitro osteogenic functions and in vivo bone tissue regeneration (Fig. 1). PCL/ PLGA/TCP scaffold was successfully fabricated using MHDS (Fig. 2). The final dimension of the scaffold was $4 \mathrm{~mm}$ in diameter and $1 \mathrm{~mm}$ in height and the line width and pore size of the scaffolds were $\sim 200$ and $300 \mu \mathrm{m}$, respectively. The surface morphology of each scaffold was investigated by scanning electron microscopy (SEM). The PCL/PLGA/TCP scaffold had a rough surface topography due to the included TCP powder (Fig. 2(a)) and collagen and bdECM were coated quite uniformly along the lines of the scaffold without blocking the pores
(Fig. 2(b) and (c)). The smoother lines of the scaffolds indicate that the collagen and bdECM coating processes have been successfully performed. The degree of collagen and bdECM coating on the scaffolds was quantified via XPS analysis (Table 1).

Size controllable 3D fabrication scaffold including diameter and pore size is the most convenient property of $3 \mathrm{D}$ printing technology. In addition, PCL/PLGA/TCP, PCL/PLGA/TCP/Col, and PCL/PLGA/TCP/bdECM scaffold have been well established in maintaining their interconnectivity. The interconnectivity of the scaffolds is important for the bone defect healing because of the host cell migration from the bone marrow and migration of the neighboring osteoblasts. ${ }^{12,18}$

\section{In vitro cell viability and proliferation results}

Cultured osteoblasts (passage \#5) isolated from neonatal rats were used to verify the attachment and proliferation behaviors

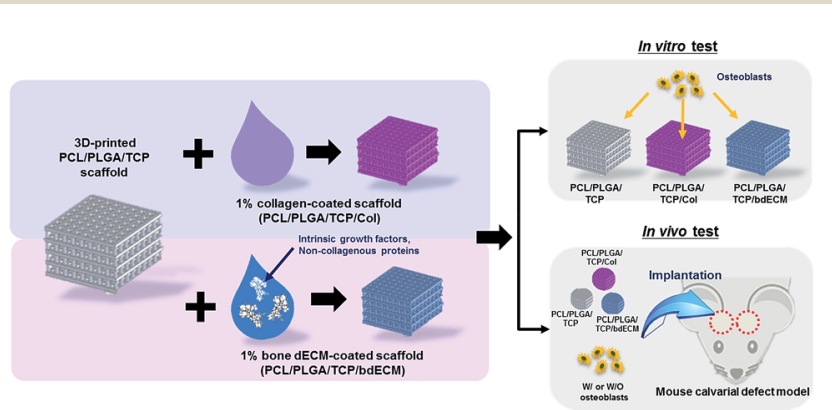

Fig. 1 Schematic elucidating the development of the bdECM coated PCL/PLGA/TCP scaffold and the experimental groups for the further in vitro and in vivo experiment.

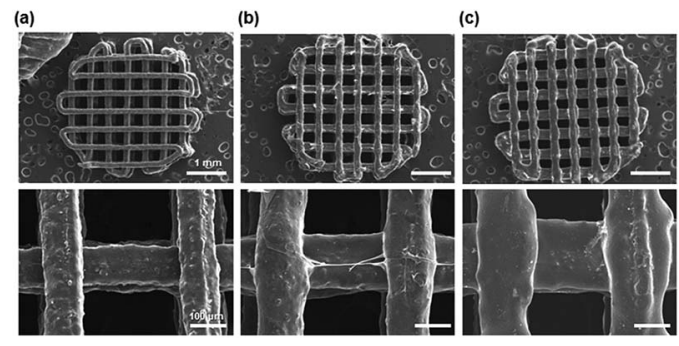

Fig. 2 SEM analysis of the 3D printed scaffolds. (a) The 3D printed PCL/PLGA/TCP scaffold showing the microperiodic structure with interconnected pores. (b) Collagen and (c) bdECM coated PCL/PLGA/ $\beta$-TCP scaffolds without blocking the pores by residual coating solution.

Table 1 Atomic component (\%) of control, collagen and bdECMcoated surfaces quantified by XPS

\begin{tabular}{llll}
\hline $\begin{array}{l}\text { Elements } \\
(\%)\end{array}$ & PCL/PLGA/ & $\begin{array}{l}\text { PCL/PLGA/ } \\
\beta-T C P / C O L\end{array}$ & $\begin{array}{l}\text { PCL/PLGA/ } \\
\beta \text {-TCP/bdECM }\end{array}$ \\
\hline $\mathrm{C}$ & $\beta-\mathrm{TCP}$ & & \\
$\mathrm{N}$ & 74.7 & 63.6 & 64.7 \\
$\mathrm{O}$ & 0 & 11.8 & 10.6 \\
& 25.3 & 24.6 & 24.7
\end{tabular}



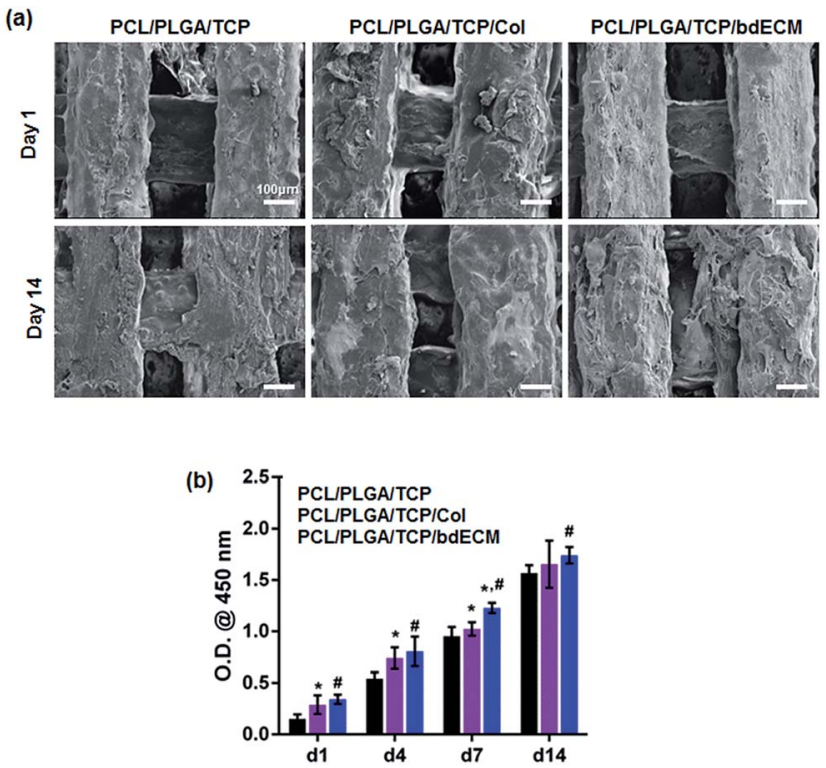

Fig. 3 Osteoblast behaviors on the bdECM-coated scaffold. (a) Adherent cells on each scaffold and growth morphology for 14 days examining by SEM analysis, and (b) the viability and proliferation trend of the cells on each scaffold $(* p<0.05$ compared to PCL/PLGA/TCP; ${ }^{\#} p<0.05$ compared to PCL/PLGA/TCP/Col).

on the fabricated scaffolds. Most adherent cells were observed on the lines of the scaffold at day 1 and grew through the pores over times (Fig. 3(a)). Interestingly, the cells on the bdECM scaffold have more aligned morphology, which is closely related to the anisotropic formation of collagen matrix and also known to contribute the mechanical properties of the bone tissue. ${ }^{15}$ Moreover, osteoblasts were more attached on the collagen or bdECM coated scaffolds than that of the noncoated scaffold, indicating that the protein-coated scaffold may enhance the cell adhesion rate. The osteoblasts proliferation ratio of all the three groups showed a similar tendency to each others (Fig. 3(b)).

The bdECM coating showed the less than approximately $30 \%$ decreased pore size of the scaffold (Fig. 2); however, bdECM coating did not interrupt the osteoblasts attachment. In contrast, bdECM coating increased the osteoblasts attachment. This result indicated that the pores of scaffold were not fully filled by bdECM, therefore osteoblasts can be migrated into the inside of the scaffold. The collagen coating obtained commercially, showed less cell attachment ratio compared to the bdECM coating. The bdECM coating provided better environment than that of collagen coating.

\section{Effect of PCL/PLGA/ $\beta$-TCP/bdECM scaffold on osteogenic function and bone mineralization}

Alkaline phosphatase (ALP), is the most widely recognized biochemical marker for the osteoblasts activity, expression of the seeded cells on each scaffold was observed by the immunofluorescence staining method. The overall area of the scaffold lines were covered with the expressed ALP marker expressed by osteoblasts, indicating that the osteoblastic activity was highly activated on the scaffolds (Fig. 4(a)). Among those three, the

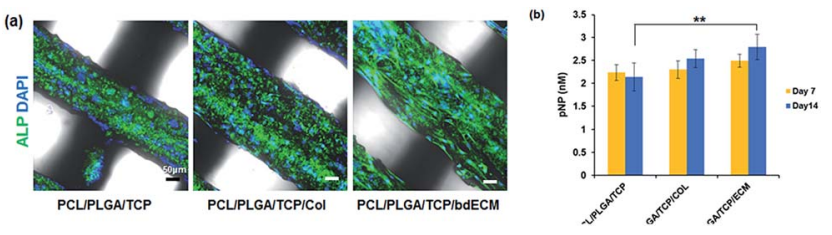

Fig. 4 ALP activity of osteoblasts on the bdECM coated scaffold. (a) ALP expression of osteoblasts on the scaffold after 7 and 14 days culture and (b) quantification amount of active ALP $(* * p<0.05)$.

cells on the bdECM coated scaffold was shown the aligned morphology and it is corresponded to the SEM analysis on day 1. Although the result of the quantitatively measured ALP activity showed that there was no significant difference between the collagen-coated scaffold and bdECM-coated scaffold, the highest activity was observed in the lysate extracted from the cells of bdECM coated scaffold at day 14 (Fig. 4(b)).

Bone mineralization by osteoblasts on the scaffold was also verified by the in vitro culture in the osteogenic differentiation media. The results demonstrated that osteoblasts on the collagen and bdECM coated scaffold deposited detectable calcium mineral after 21 days; however, larger calcium mineral amounts were presented in the bdECM coated scaffold compared to the collagen coated scaffold (Fig. 5(a)). Moreover, the colorimetric analysis results were also correlated to the staining results (Fig. 5(b)). The calcium content was consistently increased over time, and it is remarkable that the bdECM coated PCL/PLGA/TCP scaffold exhibited significantly higher calcium content than those of the other groups (Fig. 5(c)). In addition, osteogenic related mRNA expressions, including osteocalcin (OCN) and type I collagen (COL1A1), were showed higher in the bdECM coated PCL/PLGA/TCP scaffold group. This result also indicates the remarkable bone tissue regeneration capability of the bdECM coated scaffold is higher in terms of not only mineralization, but also tissue maturation and ECM synthesis (Fig. 5(d)).

Osteoblasts cultured on the bdECM coated PCL/PLGA/TCP scaffold significantly enhanced the in vitro osteogenic activity compared to the other groups. The enhanced osteogenic activity might be according to the biomolecules existing in the bdECM. bdECM contains osteogenic biomolecules such as bone morphogenetic protein-2, bone morphogenetic protein-7 and other unknown factors. ${ }^{1,2,5}$ Especially, we measured the residual amount of BMP-2 and BMP-7 in DBM and bdECM (Table 2). The concentration of BMP-2 and BMP-7 contained in the bdECM were much higher than the other groups, indicating that the higher concentration of BMP-2 and BMP-7 can improve the osteogenic activity of the PCL/PLGA/TCP/bdECM. These osteoinductive biomolecules attributed to the osteogenic differentiation of osteoblasts, also mineralization of osteoblasts. Furthermore cultured osteoblasts on the PCL/PLGA/TCP/bdECM showed the most cell adhesion ratio die to the presence of ECM. It might be various cell anchoring sites were provided by bdECM bioactive factors. ${ }^{21}$

\section{In vivo bone formation}

The in vivo bone formation efficacy was investigated using implantation of each scaffolds with or without osteoblasts onto 
(a)

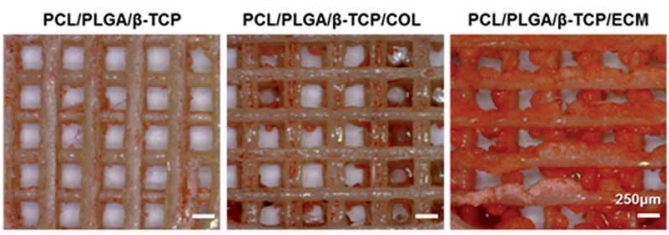

(b)

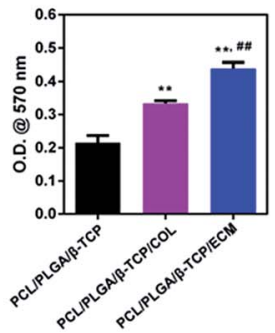

(d)

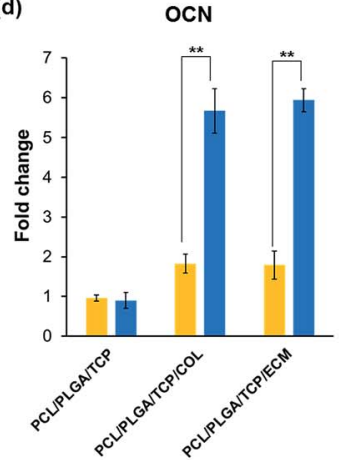

(c)

COL1A1
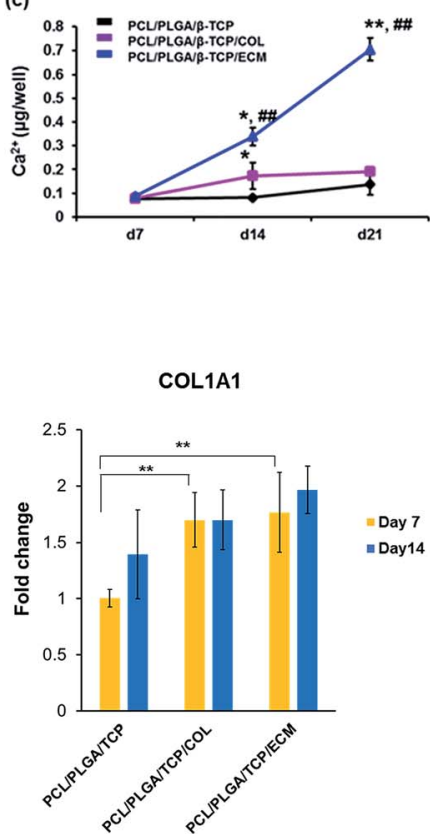

Fig. 5 Bone mineralization of osteoblasts on each scaffold. (a) Alizarin Red S staining, a marker for calcium deposition on the scaffolds at day 21 , (b) the quantification of the deposited Alizarin Red S solution on the scaffolds at the same day, and (c) calcium content at day 7, 14 and 21. (d) Oseotogenic differentiation mRNA expression (OC, right, and COL1A1, left) were quantified by qRT-PCR $(*$, \#\# and $* * p<0.05)$.

Table 2 Quantification of BMP-2 and BMP-7 in the bone powder, DBM and bdECM (BMP-2 or BMP-7 per total protein pg $\mathrm{mg}^{-1}$ )

\begin{tabular}{llrr}
\hline Target protein & Bone powder & \multicolumn{1}{c}{ DBM } & \multicolumn{1}{c}{ bdECM } \\
\hline BMP-2 & $253.1 \pm 100$ & $350.1 \pm 180$ & $1607.1 \pm 208$ \\
BMP-7 & $213.1 \pm 50$ & $200.31 \pm 110$ & $1648.62 \pm 400$
\end{tabular}

the mouse critical calvarial defect (Fig. 6(a)). The microcomputed topography (micro-CT) showed the enhanced bone regeneration by the PCL/PLGA/TCP/bdECM scaffolds implantation compared to the other scaffolds implantation. Additionally, the regenerated bone volume for the PCL/PLGA/TCP/ Col and PCL/PLGA/TCP/bdECM scaffolds implanted with osteoblasts also significantly increased compared to the scaffolds without osteoblasts (Fig. 6(b)). Histological analysis using Goldner's trichrome staining confirmed the enhanced bone regeneration efficacy of the PCL/PLGA/TCP/bdECM implantation (Fig. 7). PCL/PLGA/TCP/bdECM showed successful regenerated bone filling in the defect site including newly formed bone marrow. However, PCL/PLGA/TCP and PCL/PLGA/TCP/Col scaffold implant only showed fibrous tissues in the defect site. PCL/PLGA/TCP/Col scaffold with osteoblasts implantation resulted in the significantly enhanced bone formation area and bone density compared to the PCL/PLGA/TCP/Col scaffold without osteoblasts. Also, PCL/PLGA/TCP/bdECM implanted with osteoblasts showed enhanced bone formation area and bone density. However, PCL/PLGA/TCP implantation with osteoblasts only showed fibrous tissues similar to the PCL/ PLGA/TCP without osteoblasts.

3D printed scaffolds for this study as can be used for other type of bone tissue engineering, ${ }^{22}$ due to the controlled microstructure and interconnected porous structure potentially enhanced the host tissue ingrowth into the scaffolds implanted in the cortical sized bone defect in vivo. ${ }^{23}$ PCL/PLGA/TCP/ bdECM allowed host cells such as MSC and osteoblasts ingrowth, and cells which migrated into the scaffolds might be differentiated into the bone due to the contacting with bdECM containing biomolecules. ${ }^{24}$ The pristine 3D printed scaffolds (PCL/PLGA/TCP) prepared from synthetic artificial materials lack bone-specific bioactive signals leading to impaired bone

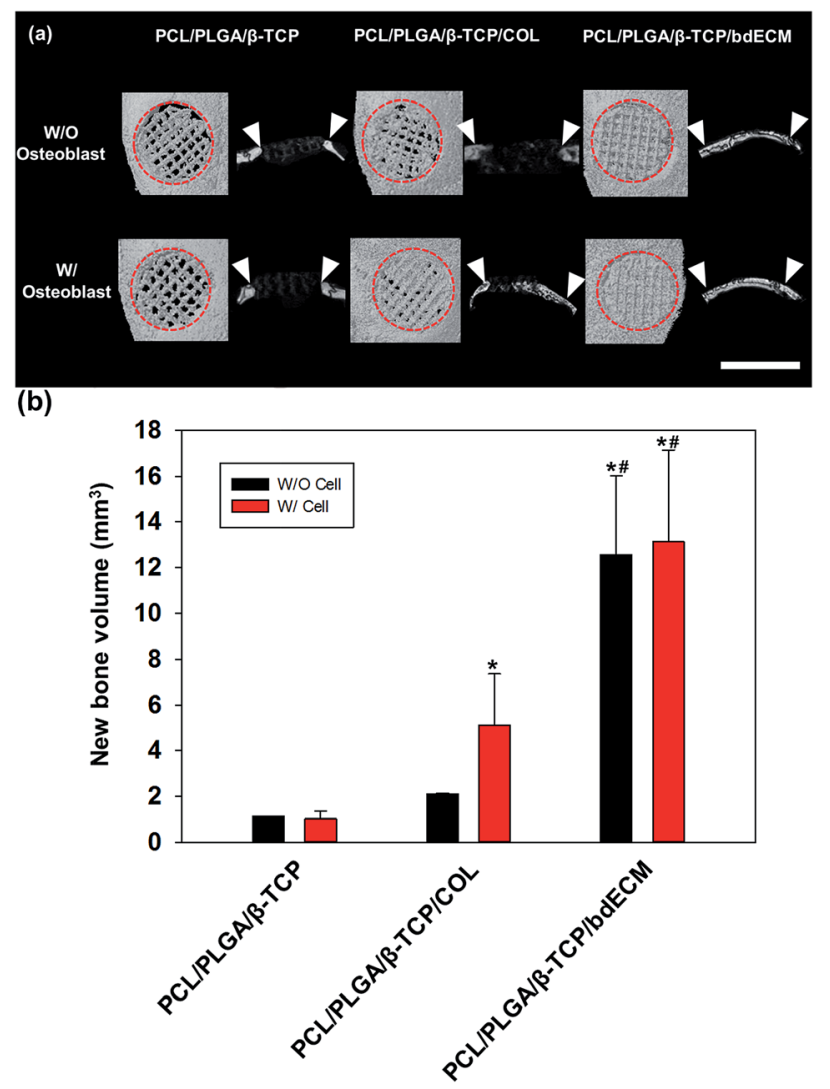

Fig. 6 Enhanced in vivo bone regeneration by bdECM coated PCL/ PLGA/TCP scaffold. Mouse calvarial defects were filled with 3 type of scaffold with or without osteoblast. (a) Bone regeneration evaluated by micro-CT. Representative micro-CT images of mouse skulls. The defects were treated with PCL/PLGA/TCP, PCL/PLGA/TCP/Col, and $\mathrm{PCL} / \mathrm{PLGA} / \mathrm{TCP} / \mathrm{bdECM}$. Scale bars $=4 \mathrm{~mm}$. (b) The volume of defect filled with neo formed bone were measured by micro-CT analysis program. ( $n=10$ defect per group). ${ }^{*} p<0.05$ compared to the $\mathrm{PCL} /$ PLGA/TCP group, " $p<0.05$ compared to the PCL/PLGA/TCP/Col group. 

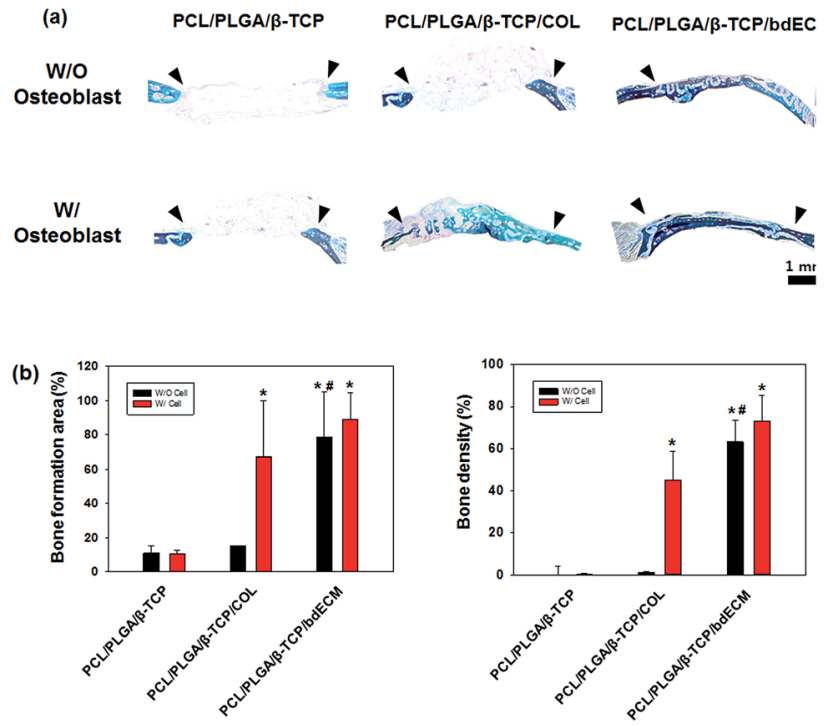

Fig. 7 Bone regeneration evaluated by histological analysis with Goldner's trichrome staining of mouse calvarial defects. Arrows indicate the defect margins. Scale bars $=2 \mathrm{~mm}$. All photographs were taken at $40 \times$ magnification. The area of new bone was determined by histomorphometric analysis ( $n=10$ defects). ${ }^{*} p<0.05$ compared to the PCL/PLGA/TCP group, ${ }^{*} p<0.05$ compared to the PCL/PLGA/TCP/ Col group

regeneration. ${ }^{25,26}$ Collagen coated scaffold PCL/PLGA/TCP/Col without osteoblasts did not show efficient bone defect healing. It might be due to the lack of osteogenic biomolecules compared to the PCL/PLGA/TCP/bdECM. The less in vitro cell adhesion ratio and osteogenic activity of collagen also affect to the in vivo healing. PCL/PLGA/TCP/bdECM with or without osteoblasts implantation showed no difference between each other. It might be sufficient amounts of host cells migrated successfully into the defect compared to that of PCL/PLGA/TCP/ Col. Thus PCL/PLGA/TCP/bdECM is not necessary of ex vivo cultured osteoblasts for critical size bone defect healing. It can reduce the side effects caused by implanted the cell. Additionally, cost and time of cell culture in the laboratory can be also reduced.

\section{Conclusions}

In this study, we demonstrated bdECM coated 3D printed scaffolds that induce in vitro osteogenic activity and in vivo critical sized bone defect healing. PCL/PLGA/TCP/bdECM showed the highest osteoblasts adhesion ratio with alignment morphology. These enhanced cell adhesion and alignment contributed the in vitro osteogenic activity of osteoblasts. Additionally, bdECM containing osteogenic biomolecules also might be contributed the in vitro osteogenic activity of osteoblasts. Furthermore, in vivo bone defect healing also enhanced compared to the other scaffolds. Interestingly, PCL/PLGA/TCP/ Col with osteoblasts showed the approximately half ratio of bone healing. However PCL/PLGA/TCP/bdECM without osteoblasts showed double times bigger bone defect healing. This result indicated that PCL/PLGA/TCP/bdECM does not require any ex vivo cultured cell implantation. Only PCL/PLGA/TCP/ bdECM is sufficient to induce the critical sized bone defect healing due to the bdECM containing biomolecules and enhanced host cell ingrowth into the defect.

\section{Acknowledgements}

This study was supported by the National Research Foundation of Korea (NRF) grant funded by the Korea government (MSIP) (No. 2010-0018294) and the Basic Science Research Program through the NRF, funded by the Ministry of Education (2014R1A1A2055348).

\section{Notes and references}

1 K. E. Benders, P. R. Weeren, S. F. Badylak, D. B. Saris, W. J. Dhert and J. Malda, Trends Biotechnol., 2013, 31, 169176.

2 R. Lomas, H. Gillan, J. Matthews, E. Ingham and J. Kearney, Biomaterials, 2001, 22, 913-921.

3 P. C. Palmer, C. J. Newcomb, S. R. Kaltz, E. D. Spoerke and S. I. Stupp, Chem. Rev., 2008, 108, 4754-4783.

4 A. George and A. Veis, Chem. Rev., 2008, 108, 4670-4693.

5 E. Gruskin, B. A. Doll, F. W. Futrell, J. P. Schmitz and J. O. Hollinger, Adv. Drug Delivery Rev., 2012, 64, 1063-1077.

6 B. Peterson, P. G. Whang, R. Iglesias, J. C. Wang and J. R. Lieberman, J. Bone Jt. Surg., Am. Vol., 2004, 86, 22432250.

7 J. C. Wang, A. Alanay, D. Mark, L. E. Kanim, P. A. Campbell, E. G. Dawson and J. R. Lieberman, Eur. Spine J., 2007, 16, 1233-1240.

8 W. G. De Long, T. A. Einhorn, K. Koval, M. McKee, W. Smith, R. Sanders and T. Watson, J. Bone Jt. Surg., Am. Vol., 2007, 89, 649-658.

9 D. C. Markel, S. T. Guthrie, B. Wu, Z. Song and P. H. Wooley, J. Inflammation Res., 2012, 5, 13-18.

10 M. Sawkins, W. Bowen, P. Dhadda, H. Markides, L. Sidney, A. Taylor, F. Rose, S. Badylak, K. Shakesheff and L. White, Acta Biomater., 2013, 9, 7865-7873.

11 Y. J. Seol, T. Y. Kang and D. W. Cho, Soft Matter, 2012, 8, 1730-1735.

12 J.-H. Shim, J.-B. Huh, J. Y. Park, Y. C. Jeon, S. S. Kang, J. Y. Kim, J. W. Rhie and D. W. Cho, Tissue Eng., Part A, 2012, 19, 317-328.

13 P. Ducheyne and K. De Groot, J. Biomed. Mater. Res., 1981, 15, 441-445.

14 J. H. Shim, J. Y. Kim, M. Park, J. Park and D. W. Cho, Biofabrication, 2011, 3, 034102.

15 M. Yazdi, S. Bernick, W. J. Paule and M. E. Nimni, Clin. Orthop. Relat. Res., 1991, 262, 281-285.

16 W. S. Pietrzak, S. N. Ali, D. Chitturi, M. Jacob and J. E. Woodell-May, Cell Tissue Banking, 2011, 12, 81-88.

17 K. Schenke-Layland, O. Vasilevski, F. Opitz, K. König, I. Riemann, K. J. Halbhuber, T. Wahlers and U. A. Stock, J. Struct. Biol., 2003, 143, 201-208. 
18 G. Chen, A. Okamura, K. Sugiyama, M. J. Wozniak, N. Kawazoe, S. Sato and T. Tateishi, J. Biomed. Mater. Res., Part B, 2009, 90, 864-872.

19 W. G. La, S. W. Kang, H. S. Yang, S. H. Bhang, S. H. Lee, J. H. Park and B. S. Kim, Artif. Organs, 2010, 34, 1150-1153.

20 J. H. Lee, C. S. Kim, K. H. Choi, U. W. Jung, J. H. Yun, S. H. Choi and K. S. Cho, Biomaterials, 2010, 31, 3512-3519. 21 P. P. Provenzano and P. J. Keely, J. Cell Sci., 2011, 124, 11951205.

22 S. Bose, S. Vahabzadeh and A. Bandyopadhyay, Mater. Today, 2013, 16, 496-501.
23 N. E. Fedorovich, E. Kuipers, D. Gawlitta, W. J. A. Dhert and J. Alblas, Tissue Eng., Part A, 2011, 17, 2473-2486.

24 W. G. La, M. Jin, S. Park, H. H. Yoon, G. J. Jeong, S. H. Bhang, H. Park, K. Char and B. S. Kim, Int. J. Nanomed., 2014, 9, 107116.

25 E. Vorndran, M. Klarner, U. Klammert, L. M. Grover, S. Patel, J. E. Barralet and U. Gbureck, Adv. Eng. Mater., 2008, 10, B67B71.

26 R. Detsch, S. Schaefer, U. Deisinger, G. Ziegler, H. Seitz and B. Leukers, J. Biomater. Appl., 2011, 26, 359-380. 